Mathias Busek*, Mario Schubert, Kaomei Guan, Frank Sonntag, Florian Schmieder, Uwe Marschner and Andreas Richter

\title{
Microphysiological system for heart tissue - going from 2D to 3D culture
}

\author{
Technological challenges and tissue integration concepts
}

\begin{abstract}
Recently, we could demonstrate positive effects of microfluidic cultivation conditions on maturation of cardiomyocytes derived from human induced pluripotent stem cells (iPS-CMs) in a 2D model. However, 3D cell culture models are much closer to physiological conditions. Combined with microfluidics, 3D systems should resemble the in-vivo conditions even better than standard 2D cultivation. For 3D models, two main technical challenges arise, the tissue integration and sufficient nutrient supply for the cells. This work focuses on concepts for the tissue integration based on a modular approach and different manufacturing technologies as well as using an oxygenator in the microfluidic device to provide sufficient oxygen supply for the cells.
\end{abstract}

Keywords: Microphysiological system, microfluidics, engineered heart tissue, oxygen control

https://doi.org/10.1515/cdbme-2019-0068

\section{Introduction}

Microphysiological systems (MPS) are a promising technology for different biological questions from basic research to applied drug screening [1]. They use microsystem technology to better mimic the cell microenvironment, ensure sufficient nutrient supply, maintain physiological medium-tocell ratios and provide specific cultivation conditions. Most of them are referred as microfluidic systems with passive components, like micro-channels, or active parts including micro-pumps and valves. We have developed a multi-layered

\section{Materials and methods}

\footnotetext{
*Corresponding author: Mathias Busek: TU Dresden, Chair of Microsystems, Nöthnitzer Str. 64, 01087 Dresden, Germany, e-mail: mathias.busek@tu-dresden.de

Mario Schubert and Kaomei Guan: TU Dresden, Institute of Pharmacology and Toxicology, Dresden, Germany.

Frank Sonntag and Florian Schmieder: Fraunhofer IWS, Dresden, Germany.

Uwe Marschner and Andreas Richter: TU Dresden, Chair of Microsystems, Dresden, Germany.
}

MPS with an integrated pneumatic micro-pump, one cell cultivation chamber, a spiral-shaped gas exchanger for oxygen control and several fluidic connectors [2].

Recently, we could successfully show that cardiomyocytes derived from human induced pluripotent stem cells (iPS-CMs) show a better maturation which comes in a line with stronger beating motion, better motion alignment, morphological changes and a higher expression of maturation related genes [3]. However, in the currently used 2D model with cardiomyocytes growing at the bottom of a cell chamber is not very close to an in-vivo-like model system of the heart. In contrast, a 3D tissue structure involving several cell types like fibroblasts or endothelial cells would much better recapitulate the heart's physiology. There are several 3D models of the heart, so-called engineered heart muscles (EHMs), presented in literature. Most of them are muscle strings or rings, fixed by flexible pillars or other retaining structures [4]. Other systems use fenestrations or porous membranes to separate the cells from direct flow but anyhow are still 2D models [5].

This work focuses on integrating a 3D model in the already developed multi-layered MPS to provide a more physiological environment for the iPS-CMs with the aim to further drive iPS-CM maturation [6]. For this, different manufacturing technologies like laser-ablation, thermal diffusion bonding, soft-lithography and plasma treatment are combined to built-up a microfluidic base chip with integrated pumps and oxygenators together with a specific cultivation chamber for EHMs generated from iPS-CMs.

\subsection{Multilayer MPS technology}

The microfluidic system consists of several laser-cut polymer foils $(250 \mu \mathrm{m}$ thick Polycarbonate (PC) sheets) bonded together by a thermal diffusion process [7]. As shown in Fig. 


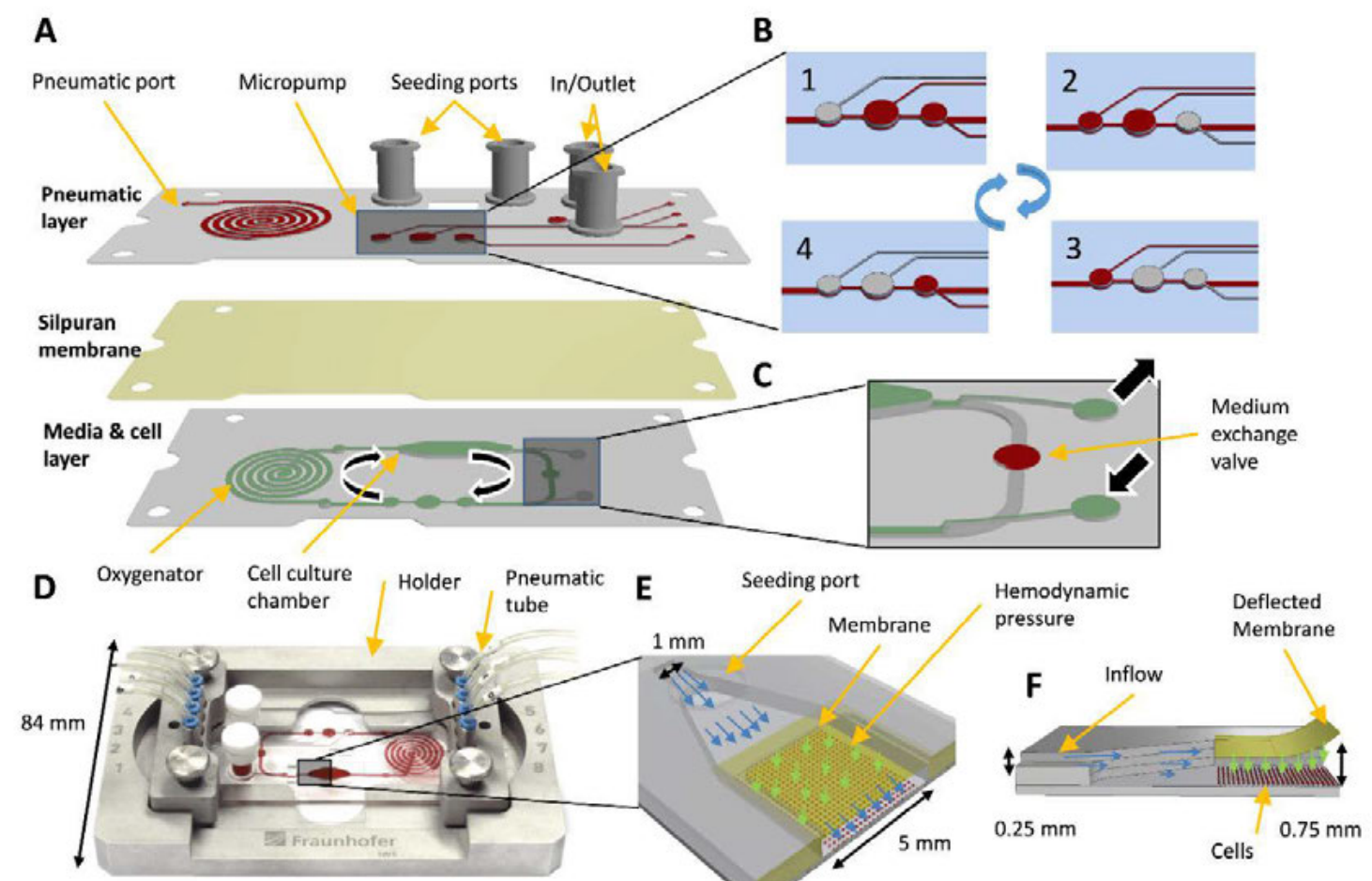

Figure 1: Views of the MPS. A) Explosion view indicating the polycarbonate foils (grey), the gas permeable membrane (yellow), the fluid (green) and the applied compressed air (red). B) Operational scheme of the pump by applying compressed air (red) or vacuum (grey) sub sequentially in 4 steps. C) By applying pressure (red) to the medium exchange valve, fluid flows from in- to outlet (black arrows) instead of in the closed circuit. D) Image of the complete device fixed in a holder. E) Perpendicular crosssection of the cell culture chamber with indicated flow profile (blue arrows), direction of hemodynamic forces (green arrows), and cell layer (red). F) Cross-sectional view of the chamber in flow direction with deflected membrane (yellow).

$1 \mathrm{~A}$, the device can be divided in a pneumatic and a fluidic part, separated by a flexible, gas-permeable silicone membrane (200 $\mu \mathrm{m}$ thick SILPURAN ${ }^{\circ}$ FILM, Wacker Chemie AG). For membrane integration, a chemical bonding process is utilized. By applying compressed air to this membrane, fluid can be moved in the fluidic part and oxygen can be exchanged at the spiral-shaped oxygenator. For both, pump and oxygenator respective gas controlling systems has been developed. This allows for precise oxygen and flow control in the chamber. As shown in Fig. 1B, fluid transport is peristaltic and an additional filling valve can be used for automatic medium exchange (Fig. 1C). Actually, cells are seeded at the bottom of the chamber shown in Fig. 1E/F and directly flushed with media on top. The yellow marked membrane allows for sufficient oxygen input during cell seeding at both seeding ports.

\subsection{Casting process for soft chamber}

Additional tissue modules e.g. for the heart can be made by casting Polydimethylsiloxane (PDMS). The high flexibility of PDMS makes it well suited to hold the muscle string in place while ensuring sufficient movability for the tissue. Moreover, PDMS has good sealing properties so the cell culture module can be directly connected to the microfluidic base device. Finally, by adding conductive fillers like carbon black, electrical pacing of the cells can be achieved. PDMS (Sylgard ${ }^{\circledR} 184$ from Dow Corning) is mixed with its curing agent in a ratio of 10:1, stirred, degassed in a vacuum oven and then poured over the master mould. To produce flexible pacing electrodes, acetylene Carbone Black powder (Alfa Aesar) is mixed with a ratio of $15 \%$ by weight to produce conductive PDMS (cPDMS) [8]. After pouring the PDMS over the moulds, it is baked at $60^{\circ} \mathrm{C}$ for several hours.

Master moulds were fabricated using a $\mathrm{CO}_{2}$ laser-cutter (Epilog Legend 36EXT) from Polymethylmethacrylate (PMMA) sheets solvent-bonded together to produce multilayered moulds with different heights.

\section{Results}

\subsection{Heart chamber design}

Currently, the cell cultivation chamber is just a channel with $5 \mathrm{~mm}$ width and $0.75 \mathrm{~mm}$ height at which the iPSC-CMs were 
cultivated under direct flow conditions. This simple channel layout has several limitations:

- Cells cannot be paced externally, so beating is based on their natural pacing rhythm.

- High contraction forces lead to detaching of the CMs as no retaining structures are included.

- Shear forces might affect cell behaviour.

- Cell-cell interactions are only partially considered; in particular, no 3D cell contacts are present.

- $\quad$ Force produced by the cells cannot be measured.

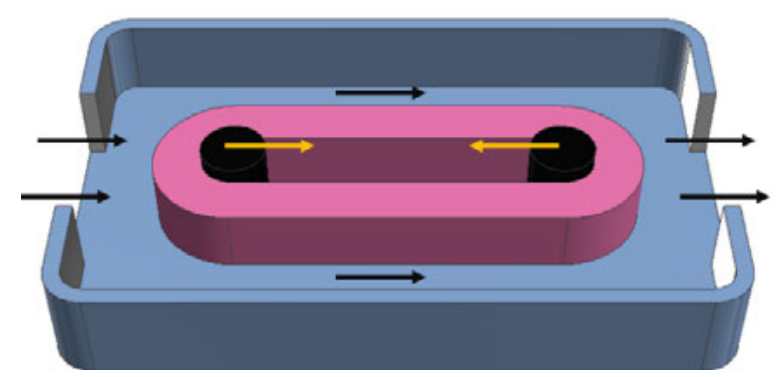

Figure 2: Chamber for mini-EHM (pink) with body made of PDMS (blue) and conductive tissue posts made of cPDMS (black). Orange arrows indicate beating motion and black arrows define flow direction.

To overcome this issue, miniaturized 3D heart muscle strings should be inserted in the cell culture chamber. For this, the culture chamber shown in Fig. 1E is opened to hold a specific cell culture module plugged in directly as shown in Fig. 2.

The black tissue posts are made of conductive cPDMS to allow pacing of the cells while holding them in place. They are going to be connected to an external pacer with needles. The modular
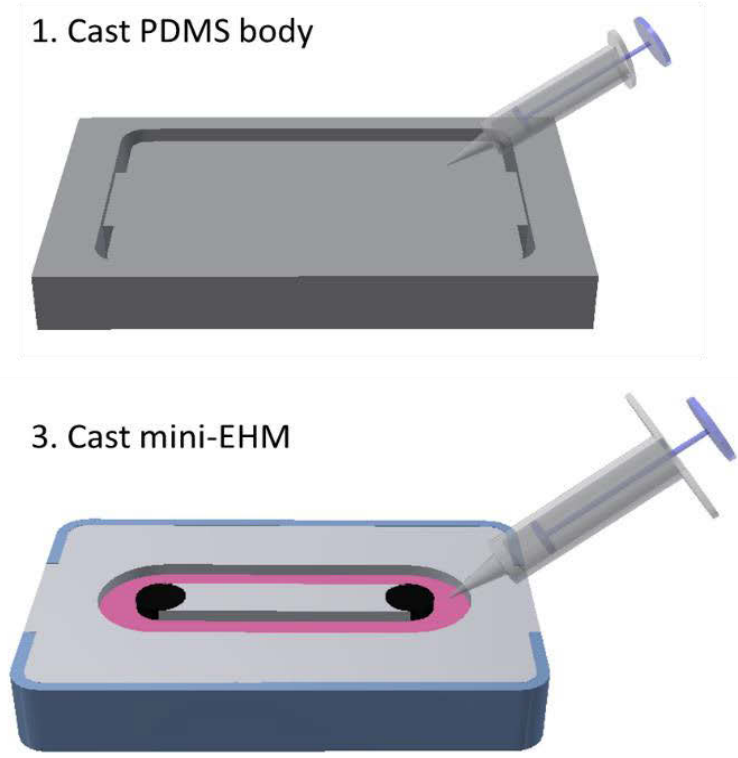

design allows static pre-cultivation of the mini-EHM before transferring it to the perfusion system. The production process (See Fig. 3) uses three different moulds, for the PDMS body, the flexible electrode made of cPDMS and the mini-EHMs. After casting, the device is transferred to the microdevice (MD) and connected to an external pacer via needle electrodes directly put into the cPDMS holders.

\subsection{Oxygen uptake and supply}

A very challenging aspect of going from a 2D model to a thick 3D tissue construct like the mini-EHM is the increased oxygen uptake by the cells. In the past, we mostly used the oxygenator shown in Fig. 1 to achieve low-oxygen environments for hypoxia studies. For this, nitrogen, carbon dioxide and compressed air was mixed and transported to the oxygenator to slowly reduce the oxygen content in the media [2]. Oxygen uptake of cells can be remodelled using a Michaelis-Menten kinetics [9], with $\mathrm{R}_{\max }$ being the maximum oxygen uptake and $\mathrm{K}_{\mathrm{M}}$ representing the Michaelis-Menten constant:

$$
\frac{\delta c_{02}}{\delta t}=-R_{\max } \cdot \frac{c_{O 2}}{K_{M}+c_{O 2}}
$$

If the amount of oxygen is greater than $5 \%$, the oxygen uptake becomes constant because cell metabolism isn't slowed down. For a $3 \mathrm{D}$ model, $\mathrm{R}_{\mathrm{EHM}}$ is now:

$$
R_{e h m}=\frac{V_{e h m}}{V_{\text {cell }}} \cdot R_{\text {cell }} \approx 4.86 \mathrm{nmol} \mathrm{min}^{-1}
$$

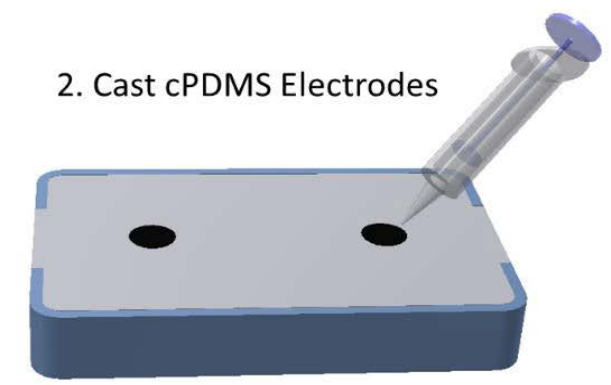

\section{Demold, insert in MD, connect to pacer}

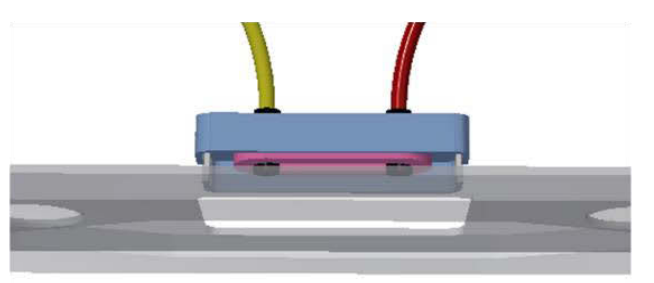

Figure 3: Heart chamber fabrication process based on casting of different materials on each other. 
Whereby, $\mathrm{V}_{\text {Енм }}$ is the volume of the muscle ring $(7.44 \mu \mathrm{L})$, $\mathrm{V}_{\text {cell }}$ is the volume of one cell $(50 \mu \mathrm{m} \cdot 10 \mu \mathrm{m} \cdot 10 \mu \mathrm{m}=5 \mathrm{pL})$ and $\mathrm{R}_{\text {cell }}$ is the oxygen uptake of one single cardiomyocyte $\left(5.44 \cdot 10^{-8} \mathrm{nmol} \mathrm{s}^{-1}\right)$ [9]. Assuming that the EHM is not fully packed with cells but also contains a significant amount of extracellular matrix this value can be further reduced.

The maximum oxygen input by the oxygenator is defined by its oxygen transfer coefficient $\mathrm{K}_{\mathrm{O} 2}$ [10]. This value depends on the geometry of the oxygenator, the mean flow velocity and its length. For a mean flow of $Q=8 \mu L \mathrm{~s}^{-1}$ and a length of $110 \mathrm{~mm}, \mathrm{~K}_{\mathrm{O} 2}$ is about 0.8 [10]. From this, the oxygen influx through the oxygenator can be calculated as follows:

$$
N_{O 2}^{\cdot}=Q \cdot K_{O 2}\left(c_{s}-c_{i n}\right)
$$

With the oxygen saturation concentration in water $\left(c_{s}=\right.$ $\left.272 \mu \mathrm{mol} \mathrm{L} \mathrm{L}^{-1}\right)$, the oxygen input becomes maximal when all oxygen is consumed by the cells $\left(c_{i n}=0\right)$. The respective influx now is: $N_{\text {max }}^{*} \approx 100 \mathrm{nmol} \mathrm{min}^{-1}$. As this value is 20 times higher than the estimated oxygen uptake of the miniEHM calculated in Eq. (2), a sufficient oxygen supply should be ensured when using the oxygenator.

\section{Summary and Outlook}

In this study, a concept for integrating a miniaturized engineered heart muscle (mini-EHM) in a microfluidic perfusion system is presented. Thereby the device is split-up in the microfluidic base device, built-up with a layer-by-layer manufacturing technology of laser-cut thermoplastic foils, and the cell culture module, which has been produced by a 2 -step casting process of PDMS and cPDMS. Finally, the EHM is casted in the soft tissue-chamber. With this approach, sufficient movability can be ensured while pacing of the cells becomes possible with the conductive cPDMS.

Beside manufacturing and designing the chamber, oxygen supply is crucial, especially when $3 \mathrm{D}$ tissue constructs are about to be cultivated in the device. For this, a helical-shaped oxygenator integrated in the microfluidic base-chip, widely used for hypoxia studies in the past, is now used to provide sufficient oxygen to the cells. To estimate whether gas exchange is sufficiently high, the oxygen exchange factor is used which has been published previously. It could be proven that the maximum oxygen influx is 20 times higher than the oxygen consumption of the mini-EHM model and therefore nutrient supply should be sufficiently high. This system offers new opportunities to culture 3D-mini EHM stuctures under hemodynamic stimulation and highly defined oxygen levels.
In future studies, this MPS will be used to address complex biological questions that require technically demanding experimental setups.

\section{Author Statement}

Research funding: The author state no funding involved. Conflict of interest: Authors state no conflict of interest. Informed consent: Informed consent has been obtained from all individuals included in this study.

\section{References}

[1] U. Marx u. a., „Biology-inspired microphysiological system approaches to solve the prediction dilemma of substance testing“, ALTEX, Bd. 33, Nr. 3, S. 272-321, Mai 2016.

[2] M. Busek, S. Grünzner, T. Steege, U. Klotzbach, und F. Sonntag, „Hypoxia-on-a-chip - Generating hypoxic conditions in microfluidic cell culture systems", Current directions in biomedical engineering, Bd. 2, Nr. Nr.1, S. 7175, Sep. 2016.

[3] M. Busek, T. Kolanowski, S. Grünzner, C. Steinfelder, K. Guan, und F. Sonntag, „Microfluidic system for enhanced cardiac tissue formation“, Current Directions in Biomedical Engineering, Bd. 3, Nr. 2, S. 367-370, 2017.

[4] S. Schaaf u. a., "Human Engineered Heart Tissue as a Versatile Tool in Basic Research and Preclinical Toxicology“, PLOS ONE, Bd. 6, Nr. 10, S. e26397, Okt. 2011.

[5] N. Huebsch u. a., „Miniaturized iPS-Cell-Derived Cardiac Muscles for Physiologically Relevant Drug Response Analyses“, Scientific Reports, Bd. 6, S. 24726, Apr. 2016.

[6] T. J. Kolanowski, C. L. Antos, und K. Guan, „Making human cardiomyocytes up to date: Derivation, maturation state and perspectives“, Int. J. Cardiol., Bd. 241, S. 379-386, 2017.

[7] U. Klotzbach, F. Sonntag, S. Günzner, M. Busek, F. Schmieder, und V. Franke, „Multilayer-based lab-on-a-chip systems for perfused cell-based assays“, Advanced Optical Technologies, Bd. 3, Nr. 5-6, S. 515-521, Dez. 2014.

[8] M. Tavakoli u. a., "Carbon doped PDMS: conductance stability over time and implications for additive manufacturing of stretchable electronics“, J. Micromech. Microeng., Bd. 27, Nr. 3, S. 035010, Feb. 2017.

[9] F. Zhao, P. Pathi, W. Grayson, Q. Xing, B. R. Locke, und T. Ma, „Effects of oxygen transport on 3-d human mesenchymal stem cell metabolic activity in perfusion and static cultures: experiments and mathematical model", Biotechnol. Prog., Bd. 21, Nr. 4, S. 1269-1280, Aug. 2005.

[10] M. Busek, S. Grünzner, T. Steege, F. Schmieder, U. Klotzbach, und F. Sonntag, „Design, characterization, and modeling of microcirculation systems with integrated oxygenators", Journal of sensors and sensor systems: JSSS, Bd. 5, Nr. Nr.1, S. 221-228, Juni 2016. 